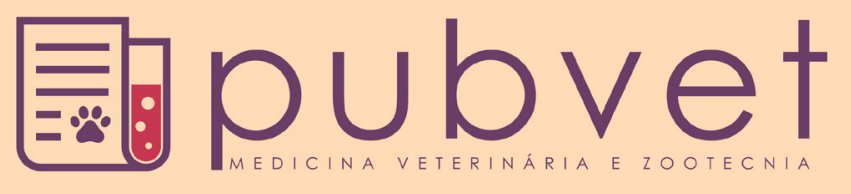

HTTP://DX.DOI.ORG/10.22256/PUBVET.V11N6.601-606

\title{
Intoxicação por ingestão de Brachiaria decumbens em bovinos no Brasil e achados patológicos decorrentes: Revisão
}

\author{
Lucilo Bioni da Fonsêca Filho ${ }^{*}$, Vitória Yuki Endo² ${ }^{2}$ Tamiris Maria da Silva ${ }^{3}$, Érica Lúcio \\ Chaves $^{4}$, Maria Luiza Alves Pereira ${ }^{5}$, Júlio Cézar dos Santos Nascimento ${ }^{6}$
}

${ }^{I}$ Discente do Curso de Graduação em Medicina Veterinária na Universidade Federal Rural de Pernambuco, Departamento de Medicina Veterinária.Recife,PE, Brasil.E-mail: lucilofilho@gmail.com

${ }^{2}$ Discente do Curso de Graduação em Medicina Veterinária na Universidade Federal Rural de Pernambuco, Departamento de Medicina Veterinária. Recife, PE, Brasil.E-mail: vitoriaye@gmail.com

${ }^{3}$ Discente do Curso de Graduação em Medicina Veterinária na Universidade Federal Rural de Pernambuco, Departamento de Medicina Veterinária.Recife, PE, Brasil.E-mail:mariatamiris92@gmail.com

${ }^{4}$ Doutoranda em Ciência Animal Tropical, Departamento de Medicina Veterinária, Laboratório de Doenças Infectocontagiosas,

Universidade Federal Rural de Pernambuco. E-mail: erica.c.l@hotmail.com.

${ }^{5}$ Discente do Curso de Graduação em Medicina Veterinária na Universidade Federal Rural de Pernambuco, Departamento de Medicina

Veterinária.Recife, PE, Brasil.E-mail: malualvesvet@gmail.com

${ }^{6}$ Professor no Centro Universitário Maurício de Nassau, Departamento de Medicina Veterinária. Recife, PE, Brasil. E-mail: juliozootecnista@hotmail.com

\begin{abstract}
RESUMO. Essa revisão de literatura tem como objetivos mostrar a importância da gramínea Brachiaria decumbens na produção animal de bovinos, abordando fatores como ganho de peso animal, palatabilidade, qualidade nutricional da gramínea, e fatores edafoclimáticos que influenciam no processo de ganho de peso. Posteriormente, incluir-seá também pontos negativos como a intoxicação decorrente do elevado consumo da gramínea, princípio tóxico, demais patologias e seus achados comumente associados em bovinos. No cenário atual, o Brasil conta com o maior rebanho comercial de bovinos de corte do mundo, e a pecuária nacional é basicamente composta de pasto, dados indicam que $80 \%$ do rebanho esteja alojado em pastagens sendo as gramíneas do gênero Brachiaria a maior fonte de alimento. A intoxicação por $B$. decumbens afeta bovinos, caprinos, ovinos, bubalinos, podendo ocorrer a qualquer época do ano, sendo a época de seca onde mais se relatam casos. Em bovinos ocorre principalmente em bezerros próximos ao desmame ou recém desmamados, a Brachiaria contém saponinas esteroidais litogênicas que induzem a formação de cristais no sistema biliar, uma das causas da doença hepática dos bovinos. Em bovinos os primeiros sinais clínicos decorrentes da intoxicação caracterizam-se por perda de apetite, depressão, anorexia, diminuição ou parada de movimentos ruminais, sialorreia e fezes secas. Por esses motivos, os bezerros não devem ser desmamados em áreas com Brachiaria decumbens, pois o estresse do desmame somado a idade do animal e a possível existência de animais geneticamente susceptíveis são alguns dos fatores cruciais para o aparecimento de alguma enfermidade.
\end{abstract}

Palavras chave: gramínea, forrageira, fotossensibilização, plantas tóxicas

\section{Brachiaria decumbens poisoning by ingestion in cattle in Brazil and pathological findings: Review}

ABSTRACT. This literature review aims to present the importance of the grass Brachiaria decumbens in the animal production of cattle, discussing factors such as animal weight gain, palatability, nutritional quality of the grass, and edaphoclimatic factors that influence the process of weight gain. Subsequently, it will also include negative points such as intoxication due to the high consumption of grass, toxic principle, other pathologies and their commonly associated findings in cattle. In the current scenario, Brazil has the largest 
commercial herd of beef cattle in the world, and the national livestock is basically pasture, data indicate that $80 \%$ of the herd is raised in pastures and the grasses of the genus Brachiaria are the largest source of food. B. decumbens intoxication affects cattle, goats, sheep, buffaloes, and can occur at any time of the year, reporting more cases in the dry season. In cattle occurs mainly in calves near weaning or recently weaned, Brachiaria contains lithogenic steroidal saponins that induce the formation of crystals in the biliary system, one of the causes of bovine liver disease. In cattle, the first clinical signs of intoxication are characterized by loss of appetite, depression, anorexia, decreased or stopped ruminal movements, sialorrhea and dry stools. For these reasons, calves should not be weaned in areas with Brachiaria decumbens, since the stress of weaning added to the age of the animal and the possible existence of genetically susceptible animals are some of the crucial factors for the appearance of some disease.

Keywords: grass, forage, photosensitization, toxic plants

\title{
Intoxicación por la ingestión de Brachiaria decumbens en ganado bovino en Brasil y sus hallazgos patológicos: Revisión
}

\begin{abstract}
RESUMEN. Esta revisión de literatura tiene como objetivo mostrar la importancia de la gramínea Brachiaria decumbens en la producción animal de bovinos, abordando factores como ganancia de peso animal, palatabilidad, calidad nutricional de la gramínea, y factores edafoclimáticos que influencian en el proceso de ganancia de peso. Posteriormente, se incluirán también puntos negativos como la intoxicación derivada del elevado consumo de la gramínea, principio tóxico, demás patologías y sus hallazgos comúnmente asociados en bovinos. En el escenario actual, Brasil cuenta con el mayor rebaño comercial de bovinos de corte del mundo, y la ganadería nacional está básicamente compuesta de pasto, datos indican que el $80 \%$ del rebaño está alojado en pastos siendo las gramíneas del género Brachiaria la mayor fuente de género Alimento. La intoxicación por $B$. decumbens afecta a bovinos, caprinos, ovinos, bubalinos, pudiendo ocurrir a cualquier época del año, siendo la época de sequía donde más se relatan casos. En bovinos ocurre principalmente en terneros próximos al destete o recién destetados, Brachiaria contiene saponinas esteroidales litogénicas que inducen la formación de cristales en el sistema biliar, una de las causas de la enfermedad hepática de los bovinos. En bovinos los primeros signos clínicos derivados de la intoxicación se caracterizan por pérdida de apetito, depresión, anorexia, disminución o parada de movimientos ruminal, sialorrea y heces secas. Por estos motivos, los becerros no deben ser destetados en áreas con Brachiaria decumbens, pues el estrés del destete sumado a la edad del animal y la posible existencia de animales genéticamente susceptibles son algunos de los factores cruciales para la aparición de alguna enfermedad.
\end{abstract}

Palabras clave: gramínea, forrajera, fotosensibilización, plantas tóxicas

\section{Introdução}

O Brasil apresenta o maior rebanho de gado de corte do mundo (ANUALPEC, 2016). Dessa maneira a pecuária extensiva tem grande importância para a economia do país (Ferraz and Felício, 2010). As pastagens presentes no país colaboram com esta atividade e em muitos casos são nativas, trazidas por colonizadores e escravos de outros continentes (Prado, 2010). Como exemplo de pastagem tem-se a Brachiaria decumbens também conhecida como capim Brachiaria seu nome popular, originária no continente Africano, mais especificamente na África do Sul, ocorrendo com mais frequência nas margens do rio Pangola e da África ocidental. Foi introduzida no Brasil pelo antigo IPEAN (Instituto de Pesquisas Experimentais Agropecuárias do Norte) atual EMBRAPA, nos anos sessenta onde apresentou boa adaptação, principalmente em áreas de cerrado. O capim Brachiaria se apresenta como uma excelente forrageira, resistente a seca e ao frio, a umidade e as pragas da lavoura como a cigarrinha das pastagens, com um ciclo vegetativo perene, além de um ciclo de vida longo e sobrevivendo por mais de um ciclo sazonal (Castro et al., 2009). O Brasil atualmente apresenta 100 milhões de hectares de pastagem cultivada, sendo 70 milhões composto do gênero Brachiaria spp e presume-se que 
aproximadamente $50 \%$ das pastagens de Brachiaria no Brasil sejam constituídas pela espécie $B$. decumbens, sendo esta a espécie mais distribuída no país. Isso se deve ao fato da $B$. decumbens ser recomendada em diversas situações como a exemplo para topografias acidentadas por apresentar facilidade em emitir quantidades maiores de estalões, apresentar fácil enraizamento com o solo favorecendo a sua cobertura e o protegendo do processo de erosão, ainda que essa característica dificulte sua combinação com outras leguminosas (Alvim et al., 2002).

Apesar de apresentar qualidades favoráveis a climas tropicais, a Brachiaria decumbens se consumida em excesso pode causar um quadro de fotossensibilização hepatogéna com também presença de edema de face e formação de crostas ao redor dos olhos, orelha, opacidade de córnea, icterícia, secreção nasal, em casos de animais mais jovens ou recém- desmamados tendem a ser mais comum a intoxicação (Saturnino et al., 2010). $\mathrm{Na}$ América do Sul, tem-se o primeiro registro de intoxicação por fotossensibilização na Venezuela, onde 12 garrotes foram a óbito durante época chuvosa, alguns achados nos animais incluíam edema de pele das orelhas e da base dos chifres, que posteriormente estendeu-se para outras partes do corpo (Döbereiner et al., 1976).

Saponinas litogênicas esteroidais são glicosídeos resultante do metabolismo secundário das plantas (Hess et al., 2003). Compostos estes formados por uma parte hidrofílica e uma parte lipofílica. $\mathrm{Na}$ identificação laboratorial de presença saponinas podem ser realizados os testes de hemólise sanguínea. Sendo que as saponinas da Brachiaria decumbens são derivadas das saponinas diosgenina e yamogenina. Existem quatro saponinas esteroidais e três sapogeninas das partes aéreas de $B$. decumbens, no rúmen a diosgenina por processo de hidrolise é reduzida em epismilagenina, esmilagenina, esmilagenona e tigogenina, e a yamogenina em episarsasapogenina sendo estas últimas as responsáveis pela formação dos cristais biliares. (Moreira et al., 2009).

Um dos problemas decorrente do depósito de cristais biliares é a fotossenbilização hepatógena, doença que pode causar uma perda econômica para o produtor uma vez que reduz o desempenho de animais acometidos, pode também leva-los a óbito em casos mais graves, já a fotossensibilização pode ser definida como um aumento na suscetibilidade da pele à luz ultravioleta que acometem principalmente animais criados a pasto. Alguns dos sinais clínicos encontrados são anorexia, diminuição ou parada dos movimentos ruminais, depressão, fezes desidratadas, animais deitados por longos períodos. Após um a dois dias os animais podem apresentar icterícia, sialorreia, urina escurecida e fotossensibilização em forma de dermatite concentrada no focinho, úbere ou em áreas de pele branca (Borges et al., 2005, Motta et al., 2000).

Borges et al. (2005) relatam em sua pesquisa fígados aumentados e mais consistentes com áreas esbranquiçadas em cinco animais, além da bile apresenta-se enegrecida e com maior volume, além do aumento da vesícula biliar, também uma severa icterícia estava presente pelo tecido subcutâneo dos animais.

\section{Revisão de literatura}

\section{Brachiaria decumbens}

Por se apresentar como uma gramínea rústica, a $B$. decumbens é amplamente utilizada no Brasil, tendo se adaptado ao clima tropical com facilidade, apresentando boa cobertura de solo, é usada para produção de forragem apesar de os pecuaristas do Brasil adotar uma forma de manejo que traz consequências como a redução no seu potencial, tanto na sua qualidade das sementes como na sua produção, dessa forma deve ser utilizado um manejo visando aumentar a produtividade e qualidade da forragem (Oliveira and Sá, 2006).

Tendo o Brasil apresentando sua maior extensão de clima tropical, a produção de forragem é caracterizada por dois períodos distintos: chuvas e seco. No período de água, a produção de forragem é favorecida, dentre outros fatores, pelas altas temperaturas, fotoperíodo longo e maior concentração de chuvas. Teixeira et al. (2011) constataram que neste período, as gramíneas do gênero Brachiaria chegam a acumular de 77 a $90 \%$ da produção total de matéria seca em relação ao período da seca; forragem que, normalmente, não é colhida de forma eficiente.

Essa inconstância na produção de forragem acarreta dificuldade em relação ao planejamento alimentar do rebanho no decorrer do ano, pois as altas taxas de lotação permitidas no período das águas são bastante reduzidas durante o período seco (Teixeira et al., 2011). 


\section{Fotossensibilização hepatógena}

Fotossensibilização pode ser definida como aumento da sensibilidade da pele à luz ultravioleta ou a ação de certas drogas, plantas ou outras substâncias, sendo uma patologia com frequência acometendo bovinos e ovinos, além de suínos, equinos, caprinos e bubalinos podendo ocorrer em qualquer época do ano. Apresenta maior número de casos em animais em processo de desmana ou recém-desmamados até dois anos de idade (Mottin et al., 2013).

De acordo com Lima et al. (2009), as saponinas são compostos oriundos do metabolismo secundário das plantas, são glicosídeos (uma classe de substâncias químicas formadas por moléculas de glúcido, glicideos, gliconas e um composto não glucidico conhecido como aglicona contendo uma ou várias cadeias de açucares, possuem seis isoprenos e trinta átomos de carbono). Saponinas são conhecidas pela formação de espuma persistente e em grande quantidade quando em meio aquoso.

De acordo com Patra and Saxena (2009) a reação de hidrólise das saponinas e sua consequente metabolização no trato digestivo dos animais vão resultar na formação de glicuronídeos, estes se ligam aos íons de cálcio originando sais insolúveis que se depositam em forma de cristais comprometendo dessa forma a excreção de metabólitos dos pigmentos de plantas do organismo. A clorofila é metabolizada a filoeritrina pelo intestino e bactérias do cólon. A insuficiência hepática para conjugar ou excretar a filoeritrina permite que esta se acumule em vasos cutâneos, onde é ativada para um estado fotodinâmico pela luz ultra-violeta.

Motta et al. (2000) afirmam que em seu estudo bovinos das raças Holandês, Charolês, Hereford, Shorthorn, cruza Charolês, cruza Shorthorn e cruza Zebu já foi observada a fotossensibilização hepatogena, alguns dos sinais clínicos incluem: anorexia, depressão, sialorreia, febre em alguns casos, lesões de fotossensibilização afetando áreas desprovidas de pelos a exemplos ao redor de olhos, focinho, vulva, períneo, úbere e áreas despigmentadas, estas lesões se apresentavam em forma de dermatite deixando à mostra áreas de tecido subcutâneo rubra e inflamadas, também podia observar edema de membros, conjuntivite com corrimento ocular purulento, cegueira em alguns casos e lesões ulcerativas na língua. Em bovinos de pele clara, gravemente afetados, as lesões podiam ser generalizadas podendo causar perda de parte da pele que se apresentava ressequida, com rachaduras e úlceras.

De acordo com Motta et al. (2000) em sua pesquisa, o curso da enfermidade variava de 7 a 20 dias e alguns animais recuperavam-se. Em quatro dos 19 surtos, não ocorreram mortes e os animais recuperaram-se após um período de até 20 dias.

Como tratamento, recomenda-se não deixar o animal exposto ao sol, sempre que possível coloca-los em piquetes de sombreamento e oferecer comida no cocho, uso de pomadas, hidratantes e protetores hepáticos, antihistamínicos. Em casos de lesões de pele, opta-se por pomadas antissépticas e cicatrizantes. Tratamentos estes com eficiência na recuperação da doença desde que a doença seja diagnosticada com o aparecimento dos primeiros sinais clínicos (Motta et al., 2000).

De acordo com Borges et al. (2005), os animais devem ser submetidos ao tratamento à base de oxitetraciclina (IM), Ivermectina (SC), pomada a base de colecalciferol, óleo de fígado de bacalhau, Zinco e Permanganato de Potássio como tratamento tópico.

\section{Achados de necropsia, lesões macroscópicas e histopatológicas}

De acordo com Costa et al. (2006) em sua pesquisa relataram quarenta e um casos de macrófagos espumosos microscopicamente, o infiltrado de macrófagos, de citoplasmas finamente vacuolares, levemente acidófilos e volumosos, com núcleos excêntricos e, frequentemente, múltiplos, apresentava-se organizado em ninhos, em grandes massas subcapsulares ou formando grandes nódulos no parênquima, em um caso foi observado colangiohepatite granulomatosa esta rica em células gigantes multinucleadas apresentando em seu centro cristais opticamente ativos.

Motta et al. (2000) descrevem em seu estudo lesões macroscópicas de 19 surtos de intoxicação nos períodos de 1984 e 1997 observou-se icterícia generalizada, fígado aumentado de tamanho, apresentando consistência firme ao corte, com coloração podendo variar de alaranjada a amarelada. Com relação à vesícula biliar, se apresentava aumentada de tamanho com conteúdo espesso e escuro. Na cavidade abdominal e torácica pôde encontrar líquido amarelado e edema do tecido subcutâneo e peritônio. Em alguns casos foi visto urina de cor escura e edema de pelve renal. Nas amostras de fígado, o órgão se 
mostrava esbranquiçado e com superfície capsular esbranquiçada e vesícula biliar distentida com conteúdo escuro e espesso.

As lesões histológicas observadas nos fígados dos bovinos estudados apresentavam uma degeneração hepatocelular, hepatócitos tumefeitos e vacuolizados, observou-se ainda células apoptóticas e megalócitos além de proliferação de tecido conjuntivo fibroso nos espaços porta. Infiltrado inflamatório, principalmente mononuclear era observado nos espaços porta e também no parênquima hepático. (Motta et al., 2000).

Lesão renal apresentando cilindros hialinos ou granulosos na luz dos túbulos da cortical e da medular, fluido proteinaceo no espaço de Bowman de alguns glomérulos, infiltrado inflamatório mononuclear foram observados distribuídos em focos na cortical e na junção corticomedular, além de congestão e degeneração das células dos túbulos da cortical e medular foram observadas em alguns animais (Motta et al., $\underline{2000}$ ).

Na pele dos bovinos, as lesões se caracterizam por serem hiperqueratosas com presenças de úlceras na epiderme e consequente formação de crostas acompanhadas de infiltrado inflamatório de polimorfonucleares e mononucleares que se distribuíam até a derme (Motta et al., 2000).

Salgado (2010) relatam no seu estudo da correlação entre presença de macrófagos espumosos e a fotossensibilização em bovinos, fígado apresentando coloração levemente esbranquiçada à amarelada podendo ocorrer aumento de consistência, de volume e de padrão lobular e afirma que tais características podem ser confundidas com outras lesões hepáticas como a esteatose e a hepatite.

\section{Considerações finais}

De acordo com o que foi abordado, pode-se dizer que a $B$. decumbens apesar de apresentar várias qualidades como boa adaptação ao clima tropical, resistência a seca e ao frio além das pragas como a cigarrinha das pastagens e boa cobertura de solo, contribuindo para reduzir os efeitos da erosão, além de ser uma gramínea com poucas exigências para cultivo deve-se ter cuidado com seu consumo excessivo principalmente em animais em processo de desmame até dois anos de idade devido ao metabolismo da planta apresentar saponinas esteroidais litogenicas e sua reação com a luz solar causar quadros de fotossensibilização hepatógena, podendo acarretar em diminuição da produtividade do animal e em casos graves óbitos, ao aparecimento dos primeiros sinais clínicos deve-se proceder com o isolamento do animal da luz solar e suspender o consumo da gramínea, optando-se por alimentar o animal diretamente no cocho.

\section{Referências Bibliográficas}

ANUALPEC. 2016. Anuário da Pecuária Brasileira, 20th edn. Instituto FNP, São Paulo, SP, Brasil.

Alvim, M. J., Botrel, M. de A. \& Xavier, D. F. 2002. As principais espécies de Brachiaria utilizadas no país. 22. ed. Juiz de Fora: Embrapa, Minas Gerais.

Borges, L., Domingues, M., Mattei, S., Miyazawa, M., Sincinetti, J., Santos, P. C. G. \& Pyles, M. D. 2005. Fotossensibilização secundária pela ingestão de Brachiária em bovino. Revista Científca Eletrônica de Medicina Veterinária, $5,1-6$.

Castro, C. R. T., Paciullo, D. S. C., Gomide, C. A. M., Muller, M. D. \& Nascimento Júnior, E. R. 2009. Características agronômicas, massa de forragem e valor nutritivo de Brachiaria decumbens em sistema silvipastoril. Pesquisa Florestal Brasileira, 60, 19-25.

Döbereiner, J., Tokarnia, C. H., Monteiro, M. C. C., Cruz, L. C. H., Carvalho, E. G. \& Primo, A. T. 1976. Intoxicação de bovinos e ovinos em pastos de Brachiaria decumbens contaminados por Pithomyces chartarum. Pesquisa Agropecuária Brasileira, 11, 87-94.

Ferraz, J. B. S. \& Felício, P. E. 2010. Production systems - An example from Brazil. Meat Science, 84, 238-243.

Hess, H. D., Kreuzer, M., Díaz, T. E., Lascano, C. E., Carulla, J. E., Soliva, C. R. \& Machmüller, A. 2003. Saponin rich tropical fruits affect fermentation and methanogenesis in faunated and defaunated rumen fluid. Animal Feed Science and Technology, 109, 79-94.

Lima, F. G., Ribeiro, C. S., Andrade, D. F., Guimarães, V. Y., Júnior, H. L. W., Haraguchi, M. \& Fioravanti, M. C. S. 2009. Braquiária: fatores que interferem nos níveis de saponina. Ciência Animal Brasileira, 1, 367-372.

Moreira, C. N., Banys, V. L., Rosa, B. C., Pinto, A. S., Silva, F. L. A., Haragushi, M. \& Fioravanti, M. C. S. 2009. Bovinos alimentados com capim Brachiaria e Andropogon: desempenho, avaliação da 
quantidade de esporos do fungo Pithomyces chartarum e teor de saponina das pastagens. Ciência Animal Brasileira, 10, 184-194.

Motta, A. C., Rivero, G. R.-C., Schild, A. L., RietCorrea, F., Mendez, M. d. C. \& Ferreira, J. L. 2000. Fotossensibilização hepatógena em bovinos no sul do Rio Grande do Sul. Ciência Rural, 30, 143-149.

Mottin, C., Prado, I. N., Maggioni, D., Eiras, C. E. \& Rivaroli, D. C. 2013. Suplementação com minerais quelatados em bovinos: uma revisão. Campo Digital. Revista de Ciências Exatas e da Terra e Ciências Agrárias, 8, 59-70.

Oliveira, S. A. \& Sá, M. E. 2006. Produção de Brachiaria decumbens em função da cultura antecessora e das adubações nitrogenada e fosfatada. Científica, 34, 178-187.

Patra, A. K. \& Saxena, J. 2009. The effect and mode of action of saponins on the microbial populations and fermentation in the rumen and ruminant production. Nutrition Research Reviews, 22, 204-219.

Prado, I. N. 2010. Produção de bovinos de corte e qualidade da carne. Eduem, Maringá, Paraná, Brasil.
Salgado, R. 2010. Foamy macrophages in the differential diagnosis of liver disease in cattle. PUBVET, 4, 1-7.

Saturnino, K. C., Mariani, T. M., BarbosaFerreira, M., Brum, K. B., Fernandes, C. E. d. S. \& Lemos, R. A. A. 2010. Intoxicação experimental por Brachiaria decumbens em ovinos confinados. Pesquisa Veterinaria Brasileira, 30, 195-202.

Teixeira, F. A., Bonomo, P., Pires, A. J. V., Silva, F., Fries, D. D. \& Hora, D. 2011. Produção anual e qualidade de pastagem de Brachiaria decumbens diferida e estratégias de adubação nitrogenada. Acta Scientiarum. Animal Sciences, 33, 241-248.

\section{Article History:}

Received 2 March 2017

Accepted 19 April 2017

Available on line 31 May 2017

License information: This is an open-access article distributed under the terms of the Creative Commons Attribution License 4.0, which permits unrestricted use, distribution, and reproduction in any medium, provided the original work is properly cited. 